\title{
A three-response task reveals how attention alters decision criteria but not appearance
}

Keith A. Schneider ${ }^{1,2,4,5, *}$, Ibrahim Malik3,5

${ }^{1}$ Department of Biology, York University, Toronto, ON, Canada

${ }^{2}$ Centre for Vision Research, York University, Toronto, ON, Canada

3Department of Psychology, York University, Toronto, ON, Canada

4Department of Psychological \& Brain Sciences, University of Delaware, Newark, DE 19716

5Center for Biological \& Brain Imaging, University of Delaware, Newark, DE 19716

*Correspondence to: keithas@udel.edu 


\begin{abstract}
Whether attention alters appearance or just changes decision criteria continues to be controversial. When subjects are forced to choose which of two equal targets, one of which has been pre-cued, has a higher contrast, they tend to choose the cued target. This has been interpreted as attention increasing the apparent contrast of the cued target. However, when subjects must decide whether the two targets have equal or unequal contrast, they respond veridically with no apparent effect of attention. The discrepancy between these comparative and equality judgments is explained by attention altering the decision criteria but not appearance. We supposed that when subjects are forced to choose which of two apparently equal targets has the higher contrast, they tend to proportion their uncertainty in favor of the cued target. To test this hypothesis, we employed a three-response task, in which subjects chose which target had the higher contrast but also had the option to report that the targets appeared equal. Across the population, this task disentangled potential attention effects on appearance from those on the decision criteria. We found that as subjects' narrowed their criteria about what constituted equal contrast, they were more likely to choose the cued target, supporting our uncertainty stealing hypothesis. Across the population. the effects of the attentional cue are explained as changes in the decision criteria and not changes in appearance.
\end{abstract}

Keywords: attention, appearance, contrast, psychophysics, vision 


\section{Introduction}

Does paying attention to a stimulus alters its appearance or merely influence the decisions we make about it? This question traces its roots to the beginnings of experimental psychology and still intrigues us today. The most recent support for the hypothesis that attention does alter appearance was provided by Carrasco, Ling, \& Read (2004). They conducted a study in which two Gabor patches on either side of fixation were presented to subjects. One of the patches was preceded by an exogenous attentional cue that compelled the subjects to covertly orient their attention to that target. The subjects then performed a comparative judgement, deciding which of the two targets appeared to have a higher luminance contrast. Carrasco et al., (2004) reported that attention seemed to increase the perceived contrast of the cued target. Unfortunately, although this result fit nicely with contrast gain model of attention that was prominent at the time (Reynolds, Pasternak, \& Desimone, 2000), it has not weathered well under the scrutiny that ensued. The problem with the comparative judgment task is that it is impossible to disentangle decision effects from real changes in apparent contrast because in the response models, the decision criterion is degenerate with apparent contrast (Schneider, 2006; Schneider \& Komlos, 2008). Changing the decision threshold has exactly the same effect on subjects' psychometric response functions as does changing the contrast. To distinguish the two, it is necessary to employ a different task.

For an alternate task, we used the equality judgment, in which subjects report whether the targets have the same or different contrast (Schneider, 2006; Schneider \& Komlos, 2008). If attention altered the contrast appearance of stimuli, then it would make two equal-contrast targets appear unequal, and it also could make two unequal targets appear equal by boosting the lower contrast target. But these two scenarios never occurred. In fact, attention had no effect at all on appearance when subjects performed the equality judgment task. In the response model for this task, the decision criterion is orthogonal to the hypothesized boost in perceived contrast due to attention. From these experiments it seemed clear that the purported attentional effects on appearance were merely an artifact of the decision process.

The main motivation for the present study is to investigate how attention influences the decision process. In the comparative judgment, the subjects' decisions 
are most susceptible to bias when they are the most uncertain and thus most difficult (Fechner, 1860) - when the attended and unattended targets are indistinguishable. When the subjects perceive the two targets as equal in contrast, they must nonetheless still choose one of the two targets. If unbiased, subjects would randomly report one or the other target as having the higher contrast, resulting in the uncertainty being evenly parcellated between the choices. However, when one target is attentionally cued, that target might be prioritized in the decision and be chosen in a larger fraction of the uncertain trials. Beck \& Schneider (2017) hypothesized that an attentional cue does not alter the appearance of its target but increases its salience and thus biases its selection.

In the present study, we sought to measure the process of uncertainty allocation between the cued and uncued targets directly, using a three-response task that has been shown to eliminate the confound between biases and perceptual effects (García-Pérez \& Alcalá-Quintana, 2012). In this task, subjects had three response options: 1. the uncued target had the higher contrast, 2. the two targets had equal contras, or 3. the cued target had the higher contrast. An additional benefit of this task is that the subjects perform the comparison and equality judgments simultaneously, instead of in separate trials, and thus cannot be criticized as differing in sensitivity, precision, difficulty or attentional strategy.

We found no significant effects of attention on appearance in this task. However, we did find that as subjects' criteria about what constituted equal contrast became narrower, they tended to allocate a greater percentage of responses to the cued target. The subjects' propensities for choosing the cued target were highly correlated with their decision criteria. In the comparative judgment task, this tendency might be interpreted as attention increasing perceived, but the addition of the equal response option allows us to see that subjects were merely disproportionately allocating a greater percentage of their responses to the cued target, when they were forced to choose between targets of apparently equal contrast.

\section{Decision mechanism and the "uncertainty stealing" hypothesis}

To analyze the three-response task, we used the same three-parameter model used to analyze the comparative and equality judgements in previous studies (Schneider, 2006; Schneider \& Bavelier, 2002; Schneider \& Komlos, 2008). The difference in contrast 
between the cued target $\left(c_{1}\right)$ and the uncued target $\left(c_{2}\right)$ is modeled as a normal distribution with mean $\alpha$, upon which a subject's decision mechanism operates. If the difference in contrast $(\Delta c)$ is less than a decision criterion $\tau$, the subject is unable to determine which contrast is higher and will respond that the two contrasts are equal $\left(c_{1}\right.$ $=c_{2}$, blue shaded region in Fig. 1A). If $\Delta c<-\tau$, the subject will judge that the uncued target had the higher contrast ( $c_{1}<c_{2}$, green shaded region in Fig. 1A), and if $\Delta c>\tau$, the subject will judge that the cued target had the higher contrast $\left(c_{1}>c_{2}\right.$, red shaded region in Fig. 1A).

Under the "uncertainty stealing" hypothesis, the attentional cue biases subjects to shift their decision criteria so that, when uncertain, they tend to choose the cued target (red hashed region, Fig. 1A). As a result, the attentional cue asymmetrically alters the window of equality, causing the cued target to be more frequently reported as having a higher contrast, and apparently shifting the mean of the underlying contrast distribution, $\alpha>0$, as shown in Figure $1 \mathrm{~B}$. Figure $1 \mathrm{C}$ shows the results of uncertainty stealing on a subject's response in the three-response task. The solid lines show a subject's natural responses uninfluenced by attention. The dashed lines show the effects of the attentional cue, changing the decision mechanism by reducing the decision criterion $\tau$ and thereby apparently increasing the mean $\alpha$ of the underlying contrast distribution. The result is that the cued target is more often reported as having a higher contrast, and the fraction of "equal contrast" responses is reduced.

In the three-response task, actual changes in contrast appearance can be distinguished from changes in the decision criterion based on the pattern of responses in the population. In Figure 2, the four plots show different possible response patterns. The green lines model the responses for which a subject indicates that the uncued target $\left(c_{2}\right)$ had a higher contrast $\left(c_{1}<c_{2}\right)$, the blue lines model the responses in which the subject perceives the two contrasts as equal $\left(c_{1}=c_{2}\right)$, and the red line models the responses for which the subject indicates the cued target $\left(c_{2}\right)$ had a higher contrast $\left(c_{1}>\right.$ $c_{2}$ ). One of the difficulties in analyzing this task across subjects is that the decision criteria can vary considerably across subjects. We recruited a moderate number (20) of subjects to take advantage of this variability by comparing across subjects the decision thresholds to the modeled boosts of attention. Figures $2 \mathrm{~A}$ and $2 \mathrm{C}$ show responses for two subjects with a liberal (Fig. 2A) and narrower (Fig. 2C) threshold for reporting 
contrast equality ( $\tau$ ), but no apparent effect of attention on apparent contrast ( $\alpha=0$ ). If attention changed appearance and not the decision criterion, we would observe a lateral shift in the responses but no changes in amplitude ( $\alpha$ and $\tau$ independent in the population), as in from Figure $2 \mathrm{~A} \rightarrow 2 \mathrm{~B}$ or $2 \mathrm{C} \rightarrow 2 \mathrm{D}$. However, if attention changed the decision criterion as in the uncertainty stealing hypothesis, then we would observe a lateral shift coupled with a decrease in amplitude due to the narrowed $\tau$ threshold, as in Figure $2 \mathrm{~A} \rightarrow 2 \mathrm{D}$ ( $\alpha$ and $\tau$ correlated in the population). Although we cannot measure this shift in individual subjects, because removing the cue would change the decision process, we can distinguish the two scenarios based on the pattern of responses across subjects. If the shifts in the comparative judgment are due to actual changes in perceived contrast, then the $\alpha$ and $\tau$ parameters should be independent in the population. However, if the shifts are due to changes in the decision criterion and not actual changes in perceived contrast, then we should expect the $\alpha$ and $\tau$ parameters to be correlated, with the subjects exhibiting large $\alpha$ also having small $\tau$.

\section{Methods}

Subjects. Thirty-two subjects (11 female), mean age 24.6 years (range 19-43), were paid to participate in the study. Two subjects were excluded after not being able to perform the practice task, and no data were collected from them. We aimed to recruit 20 subjects and kept enrolling subjects until we had 20 who passed the inclusion criterion of the fit parameter $\sigma<1$ (see below) in their data. This is a measure of the precision of their responses. Ten subjects did not meet this criterion and were excluded after their data were collected-many subjects were not performing the task and appeared to be responding randomly. Because of the unusually high number of exclusions, we show the data from all subjects, including these 10 excluded subjects, in the Appendix. All subjects had normal or corrected-to-normal visual acuity, gave their written, informed consent under a protocol approved by the York University Human Participants Review Committee, were naïve to the purpose of the experiment, and were compensated for their participation in the one-hour session (except S1, who was an author, was not compensated, and participated in 3 sessions over which the data were aggregated). 
Apparatus. The stimuli were generated on a Macintosh Pro computer (Apple, Inc., Cupertino, CA) using the Matlab (The Math Works, Inc., Natick, MA) programming language and displayed using Psychophysics Toolbox 3 functions (Brainard, 1997; Kleiner et al., 2007; Pelli, 1997) on a ViewSonic P225fb monitor (ViewSonic Corp., Walnut, CA) with a refresh rate of $119.6 \mathrm{~Hz}$. The stimuli durations reported below were constrained by the refresh rate and were rounded up to an integral multiple of the 8.4 $\mathrm{ms}$ frame rate. The output channels of the video card were combined with a video attenuator device (Video Switcher, Xiangrui Li, Los Angeles, CA) to enable 12-bit precision in the gray-scale luminance values (Li, Lu, Xu, Jin, \& Zhou, 2003). The gamma function and luminance of the monitor were measured using an LS-100 photometer (Konica Minolta Photo Imaging USA, Mahwah, NJ).

Visual stimuli. A diagram of the stimulus sequence is shown in Figure 3. Two Gabor stimuli targets, 4 cpd sine gratings, oriented vertically, zero phase and Gaussian envelopes with a standard deviation of $1^{\circ}$, were simultaneously presented for $40 \mathrm{~ms}$ on a uniform gray field. The centers of the targets were located $4^{\circ}$ eccentricity to the right or left of the fixation point. $120 \mathrm{~ms}$ before the onset of the targets, a $0.3^{\circ}$ diameter black dot was presented for $67 \mathrm{~ms}$ at the location $1.5^{\circ}$ directly above the center of one of the two targets. The luminance contrasts of the two targets were distinct and were defined as $\frac{L_{1}-L_{2}}{L_{1}+L_{2}}$, where $L_{1}$ is the maximum luminance of the sine wave component of the Gabor and $L_{2}$ is the minimum. The mean luminance of the sine wave components equaled the background luminance, $85 \mathrm{~cd} / \mathrm{m}^{2}$. The contrast of the cued target was 20,25 or $30 \%$, and the contrast of the uncued target was chosen from 21 different evenly distributed logarithmic range of contrasts spanning \pm 1 natural log units relative to the cued target contrast.

Procedure. Subjects were seated in a dark room and viewed the display unrestrained from $50 \mathrm{~cm}$. The cue stimulus appeared randomly on the left or right for each trial. Each of the 63 combinations of three contrast levels of the cued target and 21 relative contrast levels of the uncued target was repeated 20 times (6o for $\mathrm{S} 1$ across three 
sessions), for a total of 1260 trials ( 3780 for $\mathrm{S} 1$ ). The pairs of target contrasts were randomly interleaved throughout the experimental session.

The task of the subjects on each trial was to determine whether the two targets had equal contrast, or if not, which target had the higher contrast. Subjects pressed the left arrow key on the keyboard to indicate that the target on the left had the higher contrast, the up arrow to indicate that the two targets had equal contrast, or the right arrow to indicate that the target on the right had higher contrast. Subjects had unlimited time to respond. The cue stimulus for the next trial appeared $0.5^{-1} \mathrm{~s}$ after the response was made. The experimental sessions lasted approximately one hour, during which the observers were automatically allowed to rest and break fixation after every 50 trials, resuming the experiment when ready.

In pilot experiments, subjects were reluctant to use the "equal" response and therefore all subjects in the main experiment were encouraged to respond "equal" unless they were certain which target had the higher contrast. This was successful in getting subjects to use this response option. Subjects were also instructed to respond as accurately as possible, but to give their first impression, and that perseverating on their response would not help.

Each subject began the experiment with a practice run, consisting of 55 trials, with the cued contrast of $25 \%$ and five repetitions each for 11 different uncued contrasts, equally spanning the contrast range within \pm 1 natural log units relative to the cued target contrast.

Data analysis. The equivalent contrasts of the cued targets were determined by fitting the subjects' three responses to a three-parameter model combining the comparative and equality judgment models (Schneider, 2006; Schneider \& Bavelier, 2002; Schneider \& Komlos, 2008):

$$
\begin{aligned}
& P\left(c_{1}>c_{2}\right)=1-\Phi\left(\frac{\tau-\Delta c-\alpha}{\sigma}\right) \\
& P\left(c_{1}<c_{2}\right)=\Phi\left(\frac{-\tau-\Delta c-\alpha}{\sigma}\right) \\
& P\left(c_{1}=c_{2}\right)=1-P\left(c_{1}>c_{2}\right)-P\left(c_{1}<c_{2}\right)
\end{aligned}
$$


where $\Phi(x) \equiv \frac{1}{2 \pi} \int_{-\infty}^{x} e^{-u^{2} / 2} d u$ is the cumulative normal distribution, $\tau$ is the contrast difference criterion, $\Delta c$ is the actual difference in contrast between the cued target $\left(c_{1}\right)$ and the uncued target $\left(c_{2}\right), \sigma^{2}$ is the variance of the contrast difference and $\alpha$ is the hypothesized attentional boost in perceived contrast of the cued target relative to the uncued target. Each parameter has units of logarithmic contrast. The variances of the estimates of the model parameters were derived for each subject by assuming that the likelihood function is distributed in parameter space approximately normal near the optimal parameters (MacKay, 1992), and these variances were used to compute the weighted mean of each model parameter across subjects (Schneider, 2006). The perceived contrast was calculated from the model fit as $c^{\prime}=c e^{\alpha}$ for each actual contrast level $c$ of the cued target.

\section{Results}

The three-parameter model fit the three response functions well, as seen in the individual subject data in the Appendix. The modeled effect of attention on perceived contrast across subjects is summarized in Figure 4. There is an obvious trend across the three cued target contrast levels tested for the perceived contrast of the cued target to be higher than the veridical contrast, however this trend was not significant at any of the levels tested ( $p=.054$ for the $30 \%$ contrast).

The main objective of this study was to test whether the decision criterion would vary with the modeled perceived contrast, and this is shown to clearly be the case in Figure 5. There was a highly significant correlation, $r=.54, p=.0000098$, between the $\alpha$ and $\tau$ parameters, such that the higher the modeled $\alpha$, the smaller the modeled $\tau$. Subjects reporting veridical contrast ( $\alpha$ near zero) tended to have a more liberal threshold for judging the two targets as equal in contrast (larger $\tau$, on the right side of the plot in Fig. 5), whereas subjects who exhibited a greater attentional effect (larger $\alpha$ ) tended to have a stricter equivalence threshold (smaller $\tau$, left side of Fig. 5). This supports the uncertainty stealing hypothesis, that when the subjects are uncertain which target has the higher contrast, that uncertainty is preferentially parceled to the cued target response. This is a change in the decision criterion $\tau$ and does not reflect an actual change in perceived contrast caused by the cue. 


\section{Discussion}

The results of this study directly demonstrate attention changing the decision criterion and no altering perceived contrast. The subjects' decision criteria were strongly correlated with their tendency to choose the cued target, with the subjects reporting the largest effects of attention also exhibiting the strictest decision threshold. If using a simple comparative judgement test, the effects of attention on the perceived contrast could not be distinguished from changes in the decision criterion, and so such changes in the decision criterion have been mistakenly been attributed to an alteration of contrast appearance by attention (Carrasco et al., 2004).

The results of the present study are consistent with the results of previous studies that have used a equality judgment to disentangle changes in perceived contrast from changes in the decision criterion (Schneider, 2006; Schneider \& Komlos, 2008) and also studies that have explicitly measured response biases in the comparative judgment (Itthipuripat, Chang, Bong, \& Serences, 2019). This latter study did report an effect of attention on appearance at low contrasts using an equality judgment (though see Schneider, 2020). Anton-Erxleben, Abrams, \& Carrasco (2010) tried to replicate Schneider \& Komlos (2008) using equality judgments and claimed that they did show that attention increased perceived contrast, but they used a much smaller stimulus and as a result, the subjects' equality judgment psychometric curves were skewed at low contrast; when this was taken into account, the effects of attention on appearance disappeared (Schneider, 2011). Anton-Erxleben, Abrams, \& Carrasco (2011) claimed that attention still had an effect on contrast at which the psychometric functions were maximum, but Beck \& Schneider (2017) pointed out that this maximum point was still dependent on the low-contrast skew and was not an independent measure of attention.

There is considerable variation among the population in the extent of the decision bias. Some subjects are very precise in their judgements and report the veridical contrast even in the presence of an attentional cue, whereas other subjects exhibit large attentionally-driven biases. Kerzel, Zarian, Gauch, \& Buetti (2010) showed that, the less precise the subjects were in their judgments, in terms of the variance of their responses (the $\sigma$ parameter of our model and not the threshold $\tau$ ), the larger effects 
they exhibited in the comparative judgment task. They also concluded that attention changes the decision criteria and not perceived contrast.

The problem with the forced choice comparative judgment is well known that the decisions biases occur most often in trails in which the subject is uncertain, i.e. when the two stimuli look identical (Fechner, 1860), and methods have been proposed to counteract it (Jogan \& Stocker, 2014) or to explicitly model the indecision (García-Pérez \& Alcalá-Quintana, 2011). Including the third "equal" option as we have done in this study has been previously shown to allow the effects of biases and perception to be distinguished (García-Pérez \& Alcalá-Quintana, 2012).

Although early neural recordings seemed to show that attention operated through a contrast gain mechanism (Reynolds et al., 2000), and thus it was natural to assume that attention would operate similarly to increase perceived contrast, more recent neural recordings show that contrast gain is generally not the mechanism by which attention operates (Lee \& Maunsell, 2010; Williford \& Maunsell, 2006) and that attention and contrast are separable codes in visual cortex (Pooresmaeili, Poort, Thiele, \& Roelfsema, 2010). Our empirical observations that attention does not alter appearance therefore are supported by the neural recordings, and our findings support the notion that cognition in general does not affect perception (Firestone \& Scholl, 2015).

\section{Conclusions}

The results of this study demonstrate that, rather than actually increasing the perceived contrast of a cued target, attention changes the decision mechanism such that subjects, when uncertain which of two targets had the higher contrast, tend to allocate this uncertainty to the cued target. This explains why, in a comparative judgment in which subjects are forced to choose between two apparently equal targets, that the cued target is chosen more frequently, shifting the psychometric function towards the cued target even though its apparent contrast is unaltered. Since the effects of attention upon the decision mechanism cannot be distinguished from an actual change in perceived contrast using a comparative judgement, it is necessary to use an equality judgement, or to include a third "equal" response as we have done here, to accurately account for subjects' perceptions and decisions. 


\section{Acknowledgements}

The authors would like to thank Britt Anderson for suggesting this experiment, during the Attention and Conscious Perception workshop hosted by Jacob Beck and Keith Schneider at York University in Toronto, ON, Canada on May 8, 2016. 


\section{Figure captions}

Figure 1. Decision mechanism and "uncertainty stealing". A. The difference in contrast between the cued target $\left(c_{1}\right)$ and the uncued target $\left(c_{2}\right)$ is modeled as a normal distribution with mean $\alpha$, upon which a subject's decision mechanism operates. If the difference in contrast $(\Delta c)$ is less than a decision criterion $\tau$, the subject is unable to determine which contrast is higher and will respond that the two contrasts are equal $\left(c_{1}\right.$ $=c_{2}$, blue shaded region). If $\Delta c<-\tau$, the subject will judge that the uncued target had the higher contrast ( $c_{1}<c_{2}$, green shaded region), and if $\Delta c>\tau$, the subject will judge that the cued target had the higher contrast ( $c_{1}>c_{2}$, red shaded region). Under the uncertainty stealing hypothesis, the attentional cue biases subjects' decision criteria so that, when uncertain, they tend to choose the cued target (red hashed region). B. The attentional cue asymmetrically shift the window of equality, causing the cued target to be more frequently reported as having a higher contrast, and apparently shifting the mean of the underlying contrast distribution, $\alpha>0$. C. The results of uncertainty stealing on a subject's response in the three-response task. The solid lines show a subject's natural responses uninfluenced by attention. The dashed lines show the effects of the attentional cue, changing the decision mechanism by reducing the decision criterion $\tau$ and thereby apparently increasing the mean $\alpha$ of the underlying contrast distribution. The result is that the cued target is more often reported as having a higher contrast, and the fraction of "equal contrast" responses is reduced.

Figure 2. Models of response possibilities. The four plots show different possible response patterns. The green lines model the responses for which a subject indicates that the uncued target $\left(c_{2}\right)$ had a higher contrast $\left(c_{1}<c_{2}\right)$, the blue lines model the responses in which the subject perceives the two contrasts as equal $\left(c_{1}=c_{2}\right)$, and the red line models the responses for which the subject indicates the cued target $\left(c_{2}\right)$ had a higher contrast $\left(c_{1}>c_{2}\right)$. Different subjects naturally have a different decision criterion. A and $C$ show responses for two subjects with a liberal (A) and narrower (C) threshold for reporting contrast equality $(\tau)$, but no apparent effect of attention on apparent contrast $(\alpha=0$ ). If attention changes appearance and not the decision criterion, then we would observe a lateral shift in the responses but no changes in amplitude ( $\alpha$ and $\tau$ independent in the population), as in from $\mathrm{A} \rightarrow \mathrm{B}$ or $\mathrm{C} \rightarrow \mathrm{D}$. However, if attention 
changes the decision criterion as in the uncertainty stealing hypothesis, then we would observe a lateral shift and also a change in amplitude, as in $\mathrm{A} \rightarrow \mathrm{D}$ ( $\alpha$ and $\tau$ correlated in the population).

Figure 3. Stimulus sequence. Subjects fixated for 500-1000 ms, after which a cue appeared at $4^{\circ}$ eccentricity on the left or right. The cue disappeared after $67 \mathrm{~ms}$, and $120 \mathrm{~ms}$ after the cue onset, two Gabor grating stimuli appeared centered at the same eccentricity, one of which was slightly below the location where the cue had appeared. After $40 \mathrm{~ms}$, the stimuli disappeared, and subjects reported their relative contrasts.

Figure 4. Equivalent contrast. The perceived contrast is plotted as a function of actual contrast, $c^{\prime}=c e^{\alpha}$. Each $\times$ symbol represents a single subject. The solid line is the perceived contrast calculated from the weighted average of the $\alpha$ parameters across subjects. The shaded region shows the extent of the $95 \%$ confidence interval.

Figure 5. $\alpha$ and $\tau$ correlation. Each dot represents the $\alpha$ and $\tau$ parameters derived from the fit to a single subject and single cued target contrast. The dashed line is the linear fit. The correlation was $r=.54, p=.0000098$.

Appendix. The individual data for all subjects are shown. The three plots in each row belong to the same subject. The three columns represent the three different cued target contrasts $(20,25$ or $30 \%)$. The filled circles plot the probability of each response for each cued target contrast $\left(c_{1}\right)$ and uncued target contrast $\left(c_{2}\right)$, and the solid lines are the three-response model fits. The fit parameters are shown at the top of each sub-plot. The first 20 subjects shown were included in the study, and the last 10 subjects were excluded, as they did not meet the inclusion criterion of $\sigma<1$ for any of their model fits (see Methods). 


\section{References}

Anton-Erxleben, K., Abrams, J., \& Carrasco, M. (2010). Evaluating comparative and equality judgments in contrast perception: attention alters appearance. Journal of Vision, 1O(11), 6, 1-22. https://doi.org/10.1167/10.11.6

Anton-Erxleben, K., Abrams, J., \& Carrasco, M. (2011). Equality judgments cannot distinguish between attention effects on appearance and criterion: A reply to Schneider (2011). Journal of Vision, 11(13), 8-8. https://doi.org/10.1167/11.13.8

Beck, J., \& Schneider, K. A. (2017). Attention and Mental Primer. Mind \& Language, 32(4), 463-494. https://doi.org/10.1111/mila.12148

Brainard, D. H. (1997). The Psychophysics Toolbox. Spatial Vision, 1O(4), 433-436. https://doi.org/10.1163/156856897Xoo357

Carrasco, M., Ling, S., \& Read, S. (2004). Attention alters appearance. Nature Neuroscience, 7(3), 308-13. https://doi.org/10.1167/12.9.1387

Fechner, G. T. (1860). Elemente der Psychophysik. Leipzig: Breitkopf und Härtel.

Firestone, C., \& Scholl, B. J. (2015). Cognition does not affect perception: Evaluating the evidence for top-down effects. Behavioral and Brain Sciences, 39.

https://doi.org/10.1017/S0140525X15000965

García-Pérez, M. A., \& Alcalá-Quintana, R. (2011). Improving the estimation of psychometric functions in 2AFC discrimination tasks. Frontiers in Psychology, 2(MAY), 1-9. https://doi.org/10.3389/fpsyg.2011.00096

García-Pérez, M. A., \& Alcalá-Quintana, R. (2012). Shifts of the psychometric function: Distinguishing bias from perceptual effects. Quarterly Journal of Experimental Psychology, (January 2014), 1-19. https://doi.org/10.1080/17470218.2012.708761

Itthipuripat, S., Chang, K.-Y., Bong, A., \& Serences, J. T. (2019). Stimulus visibility and uncertainty mediate the influence of attention on response bias and visual contrast appearance. Journal of Vision, 19(14), 8. https://doi.org/10.1167/19.14.8

Jogan, M., \& Stocker, A. A. (2014). A new two-alternative forced choice method for the unbiased characterization of perceptual bias and discriminability. Journal of Vision, 14(3), 1-18. https://doi.org/10.1167/14.3.20

Kerzel, D., Zarian, L., Gauch, A., \& Buetti, S. (2010). Large effects of peripheral cues on appearance correlate with low precision. Journal of Vision, 1O(11), 26. https://doi.org/10.1167/10.11.26 
Kleiner, M., Brainard, D., Pelli, D., Ingling, A., Murray, R., \& Broussard, C. (2007). Perception. Perception (Vol. 36). [Pion Ltd.].

Lee, J., \& Maunsell, J. H. R. (2010). The Effect of Attention on Neuronal Responses to High and Low Contrast Stimuli. Journal of Neurophysiology, 104(2), 960-971. https://doi.org/10.1152/jn.01019.2009

Li, X., Lu, Z.-L., Xu, P., Jin, J., \& Zhou, Y. (2003). Generating high gray-level resolution monochrome displays with conventional computer graphics cards and color monitors. Journal of Neuroscience Methods, 130(1), 9-18. https://doi.org/10.1016/So165-0270(03)00174-2

MacKay, D. J. C. (1992). Bayesian interpolation. Neural Computation, 4(3), 415-447.

Pelli, D. G. (1997). The VideoToolbox software for visual psychophysics: transforming numbers into movies. Spatial Vision, 10(4), 437-442. https://doi.org/10.1163/156856897Х00366

Pooresmaeili, A., Poort, J., Thiele, A., \& Roelfsema, P. R. (2010). Separable codes for attention and luminance contrast in the primary visual cortex. Journal of Neuroscience, 3o(38), 12701-11. https://doi.org/30/38/12701 [pii] 10.1523/JNEUROSCI.1388-10.2010

Reynolds, J. H., Pasternak, T., \& Desimone, R. (2000). Attention increases sensitivity of V4 neurons. Neuron, 26(3), 703-14. https://doi.org/10.1016/So8966273(00)81206-4

Schneider, K. A. (2006). Does attention alter appearance? Perception \& Psychophysics, 68(5), 800-814. https://doi.org/10.3758/BFo3193703

Schneider, K. A. (2011). Attention alters decision criteria but not appearance: a reanalysis of Anton-Erxleben, Abrams, and Carrasco (2010). Journal of Vision, 11(13), 7. https://doi.org/10.1167/11.13.7

Schneider, K. A. (2020). A note on the equality judgment experiment in Itthipuripat, et al. (2019). PsyArXiv. https://doi.org/10.31234/osf.io/kbhvg

Schneider, K. A., \& Bavelier, D. (2002). Components of visual prior entry. Journal of Vision, 2(7). https://doi.org/10.1167/2.7.439

Schneider, K. A., \& Komlos, M. (2008). Attention biases decisions but does not alter appearance. Journal of Vision, 8(15), 3, 1-10. https://doi.org/10.1167/8.6.1094

Williford, T., \& Maunsell, J. H. R. (2006). Effects of Spatial Attention on Contrast 
Response Functions in Macaque Area V4. Journal of Neurophysiology, 96, 40-54. https://doi.org/10.1152/jn.01207.2005. 
Figures

Figure 1
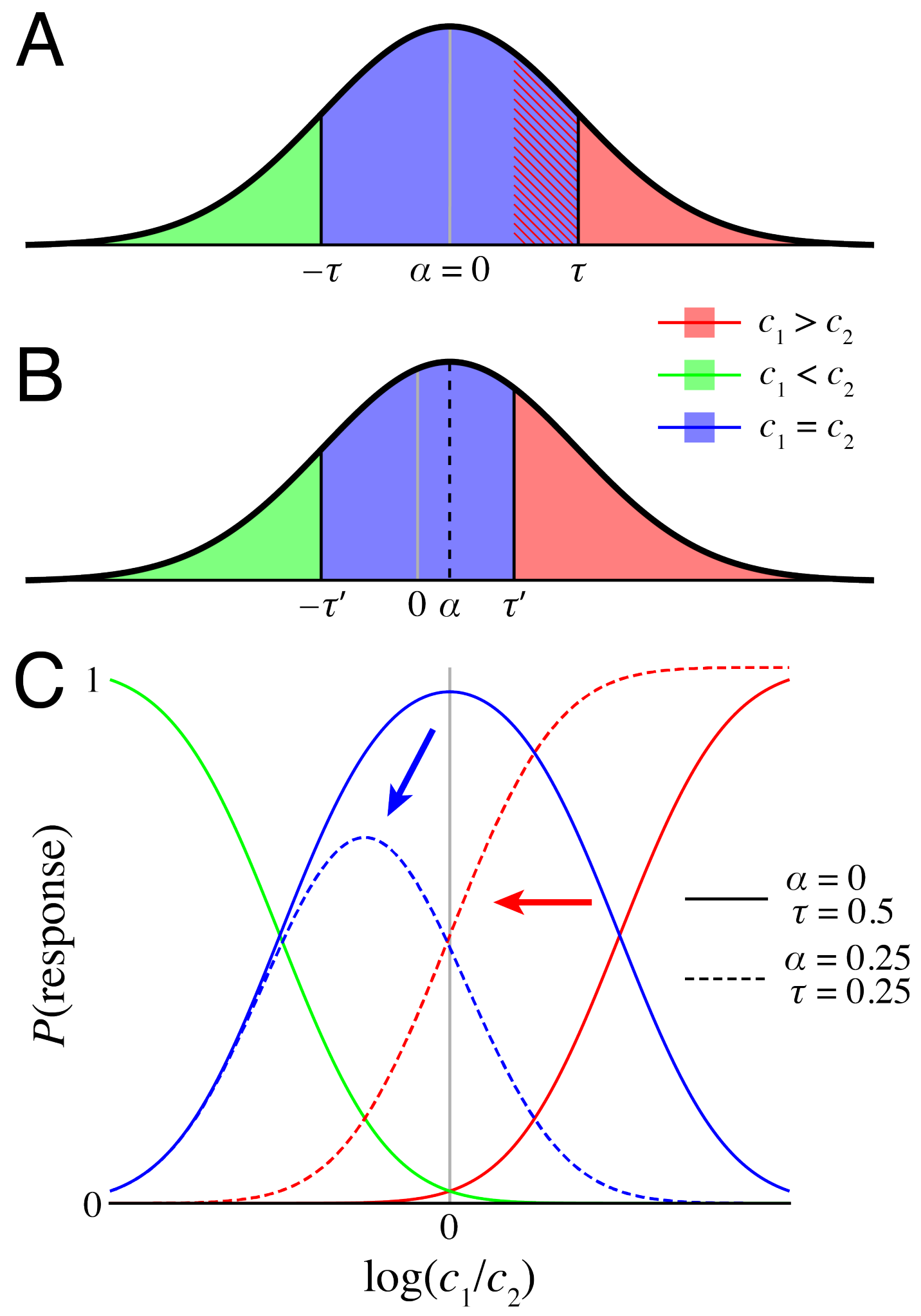
Figure 2
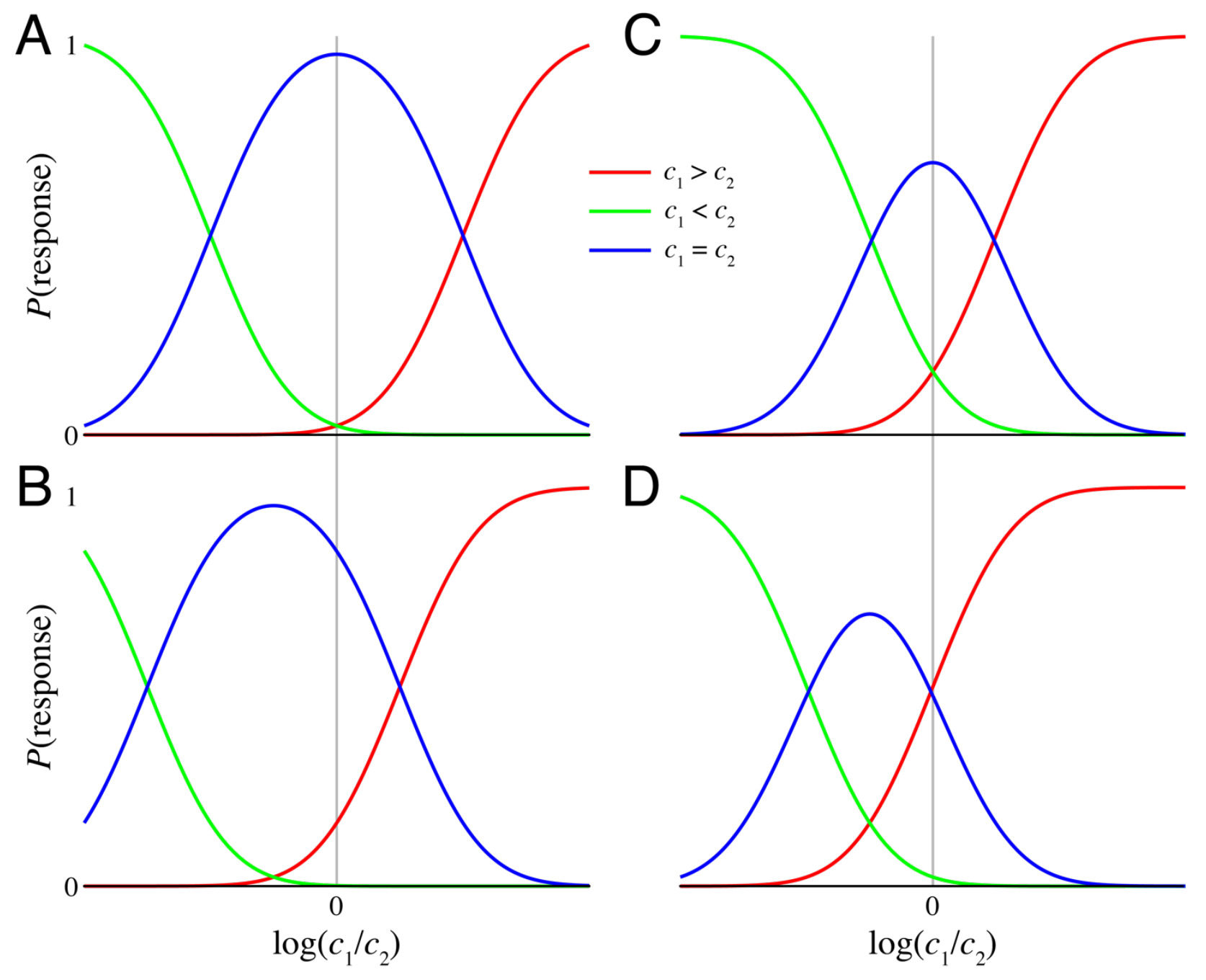
Figure 3

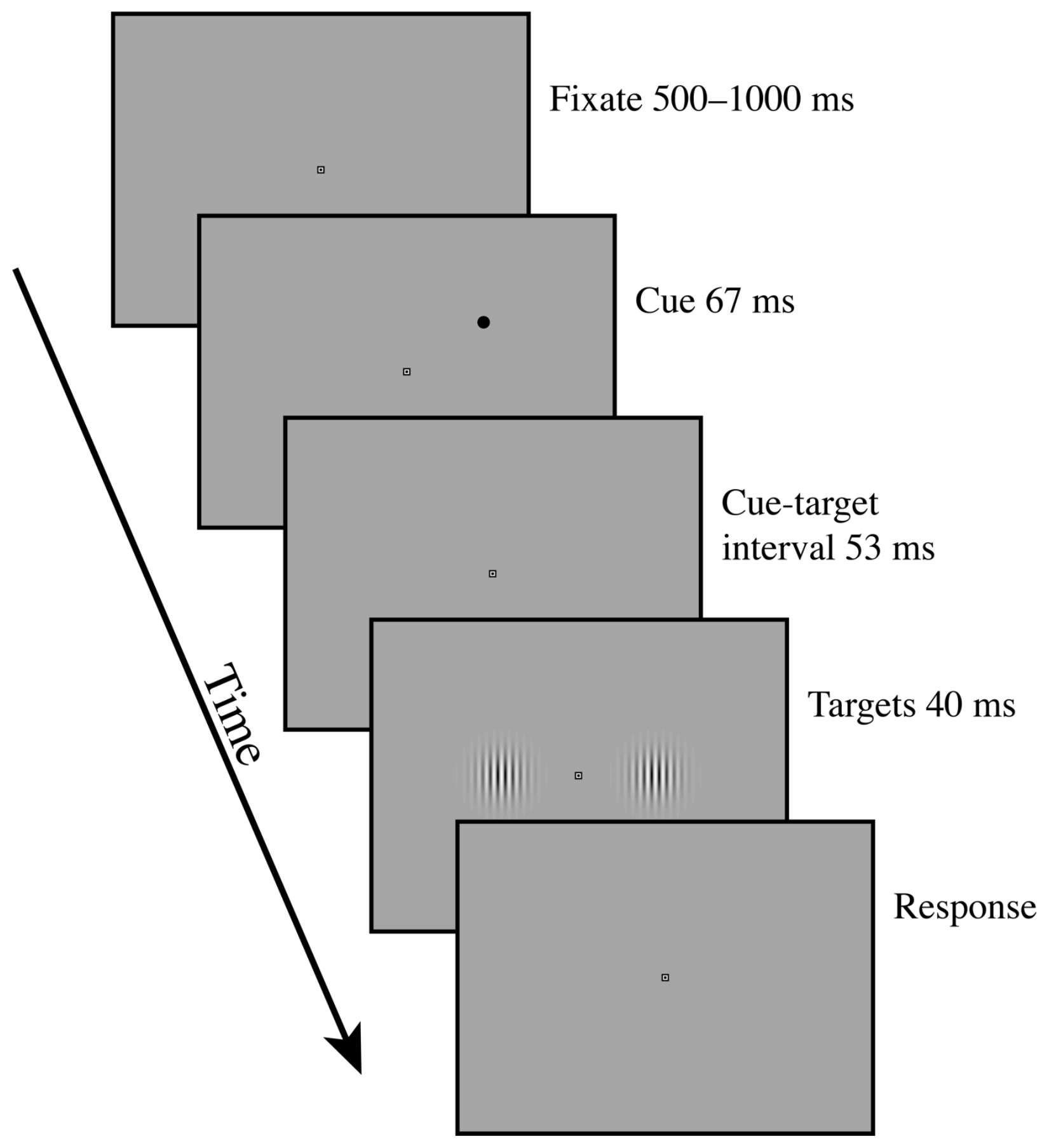


Figure 4

45-

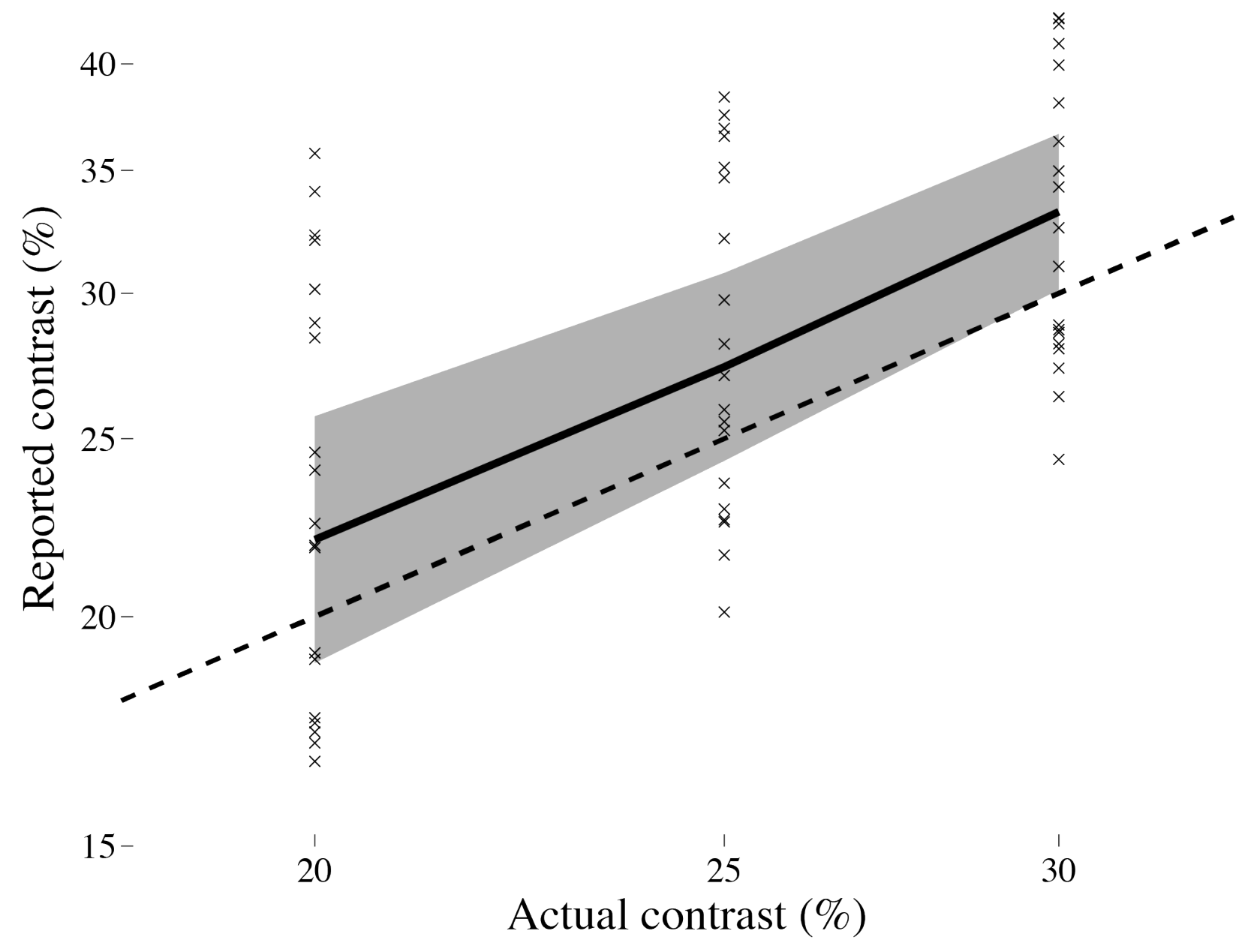


Figure 5

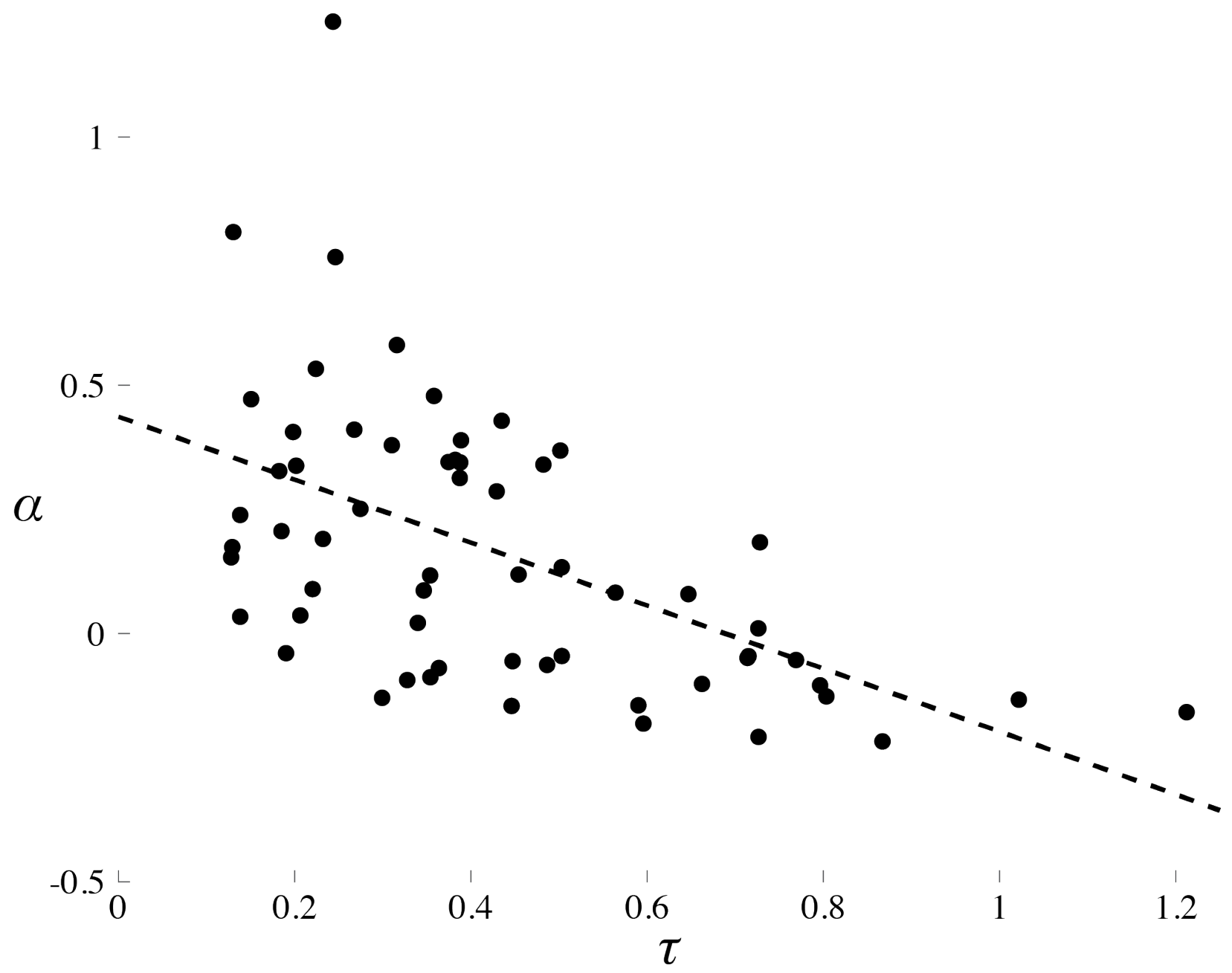


Appendix: Individual subject data

$20 \%$

S1

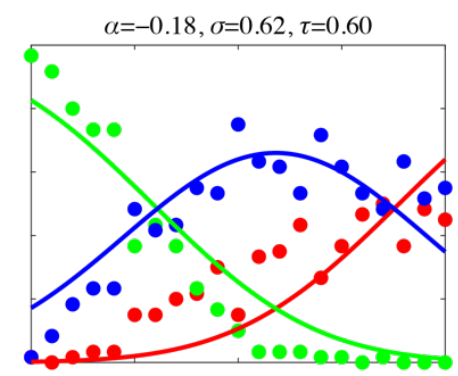

S4
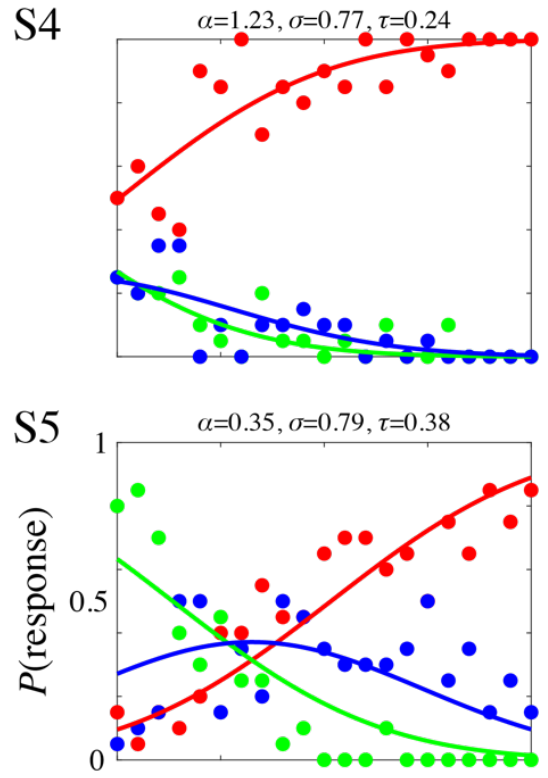

S6

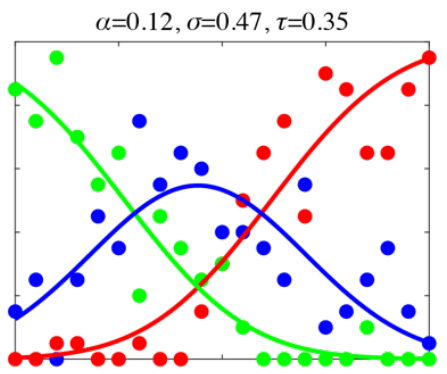

S7

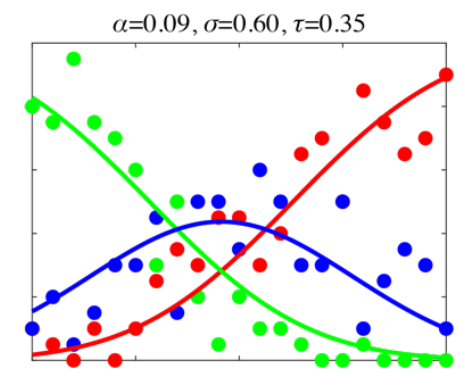

$25 \%$
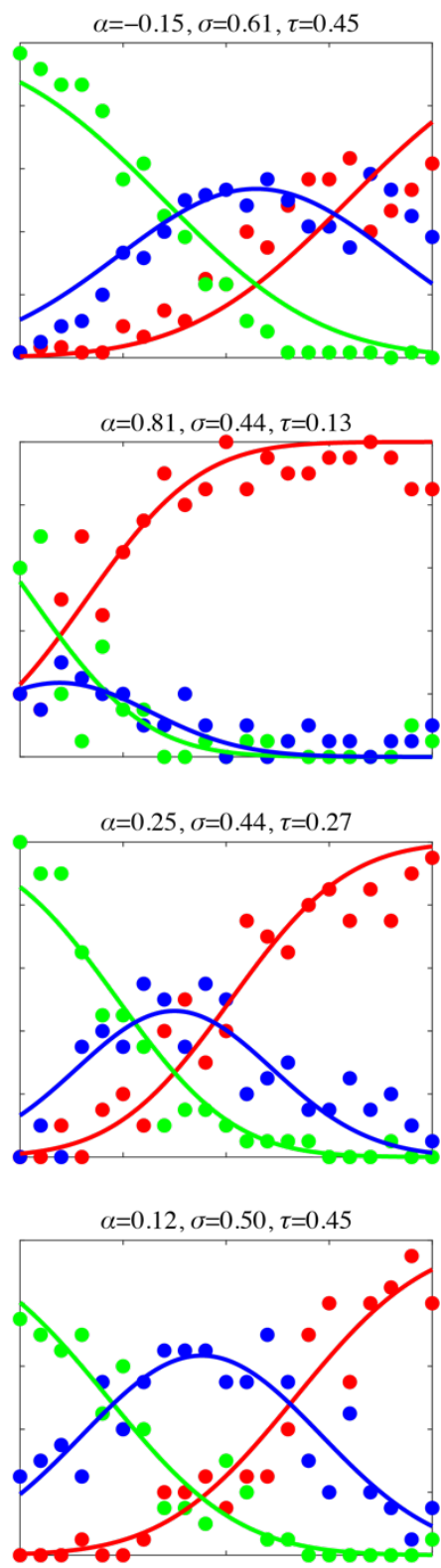

$\alpha=0.02, \sigma=0.53, \tau=0.34$

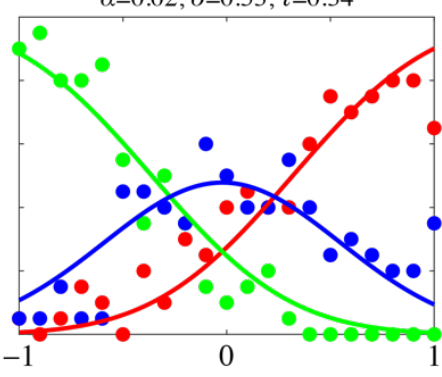

$\log \left(c_{1} / c_{2}\right)$
$30 \%$
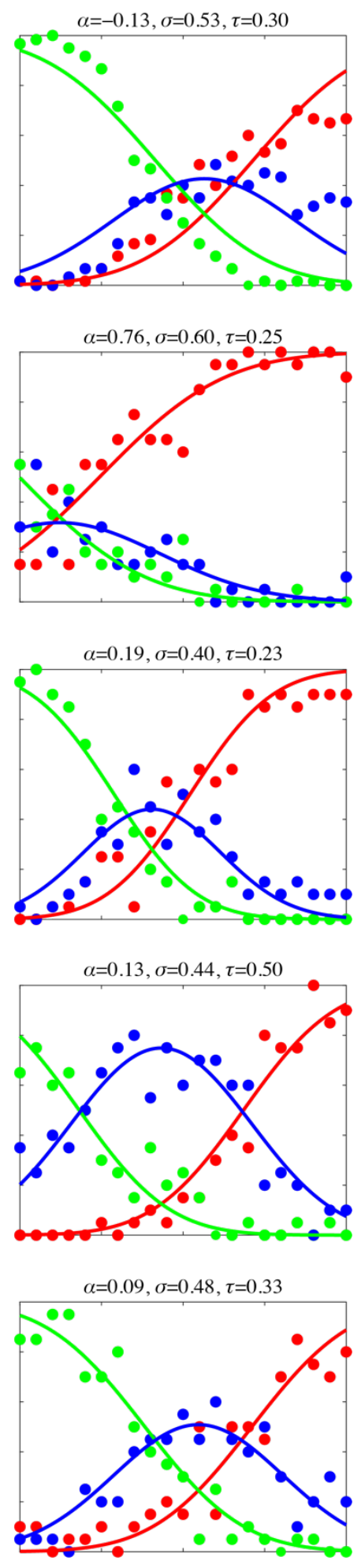
$20 \%$

S8

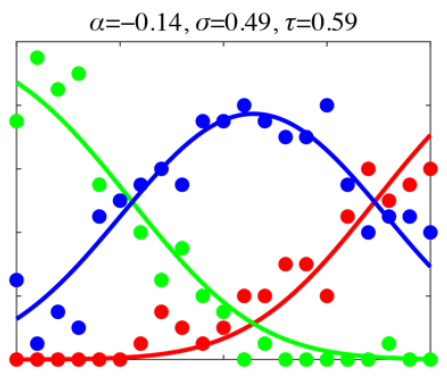

S9

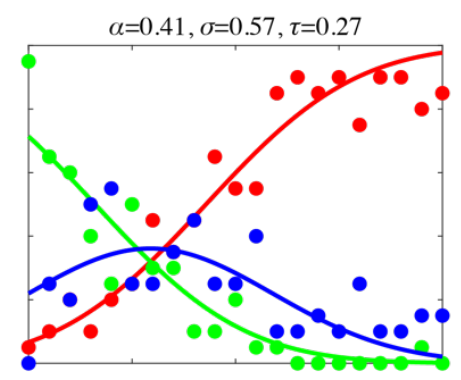

S11

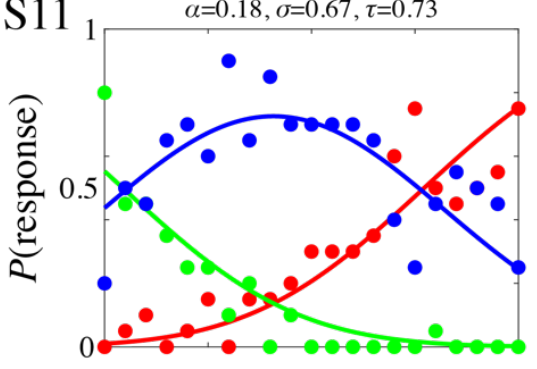

S12

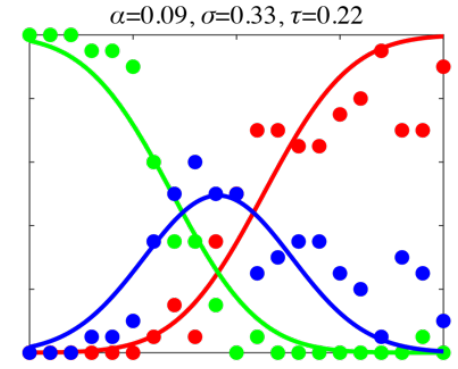

S13

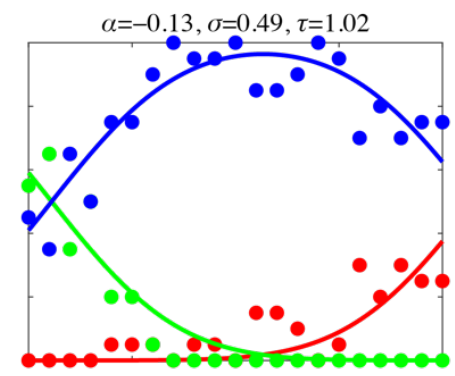

$25 \%$
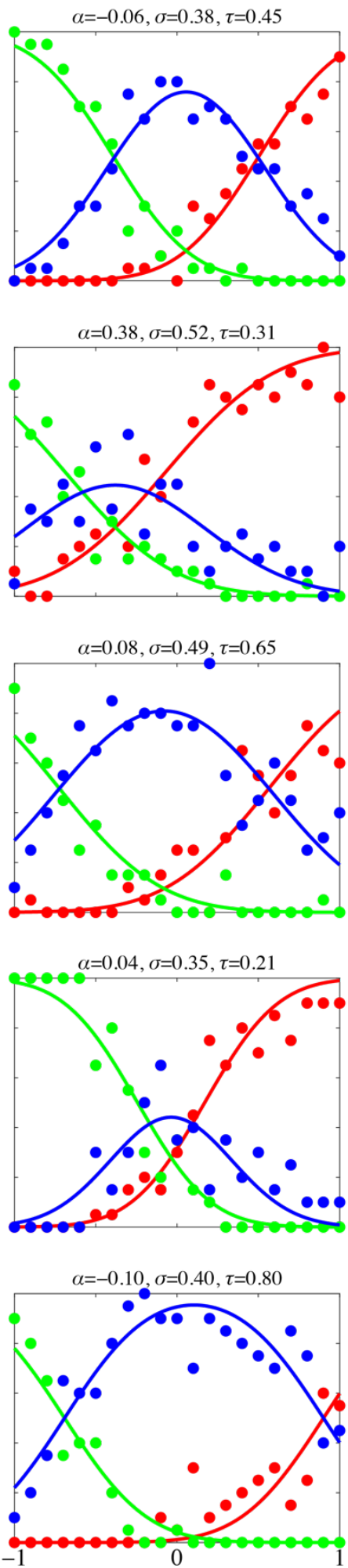

$\log \left(c_{1} / c_{2}\right)$
$30 \%$
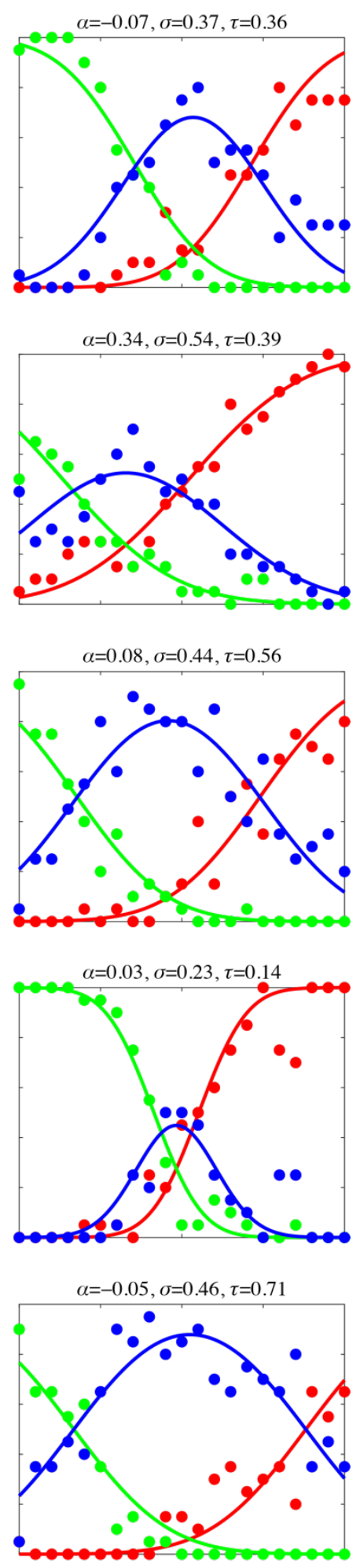
$20 \%$

S14

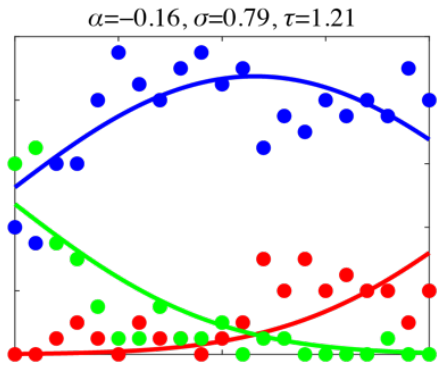

S15

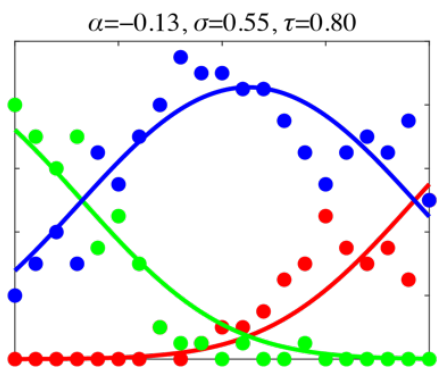

S16

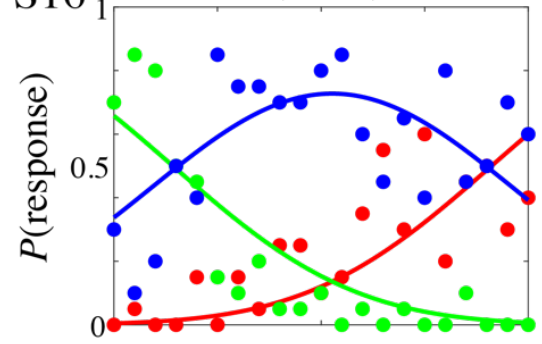

S17

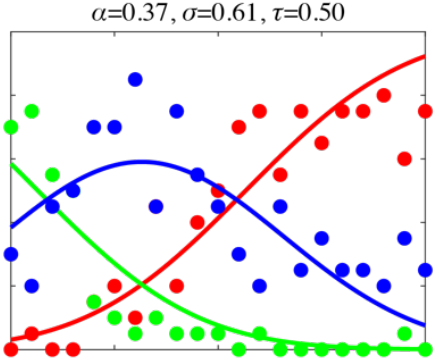

S18

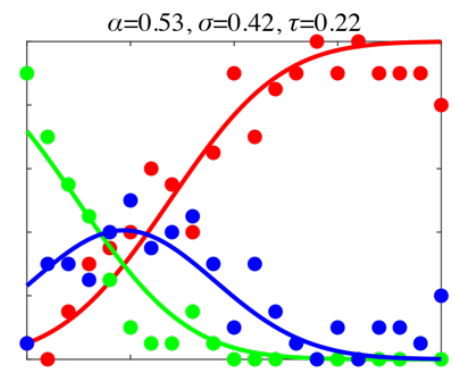

$25 \%$

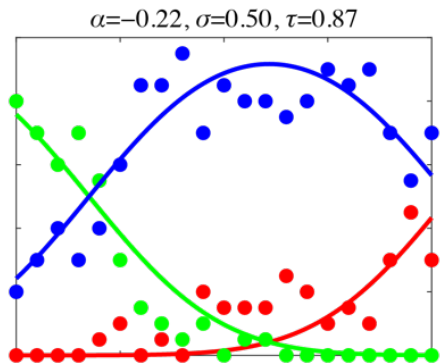

$\alpha=0.01, \sigma=0.55, \tau=0.73$
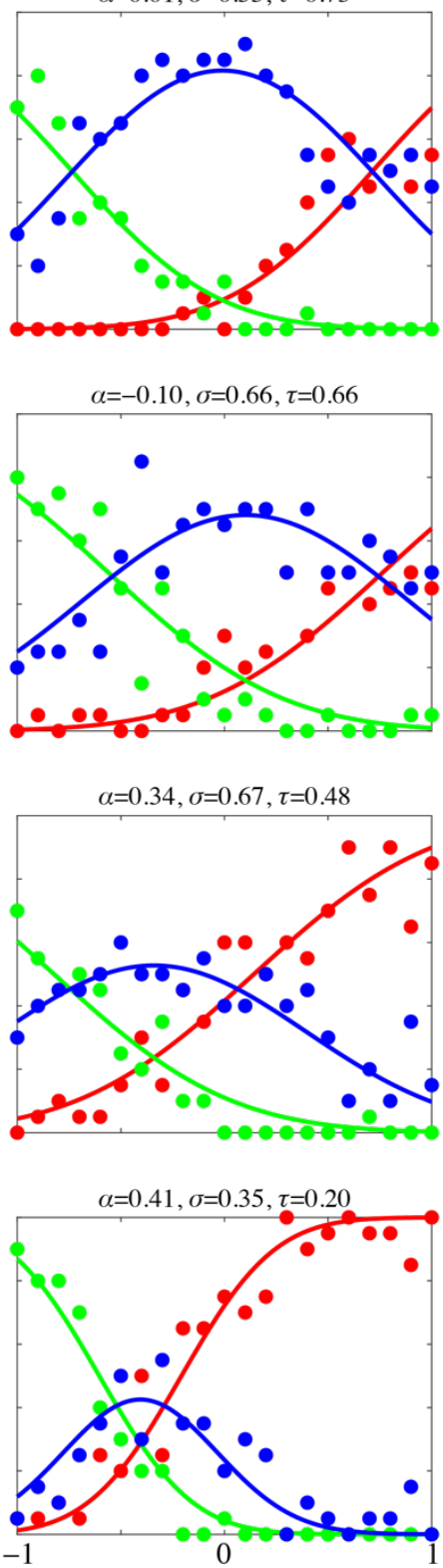

$\log \left(c_{1} / c_{2}\right)$
$30 \%$
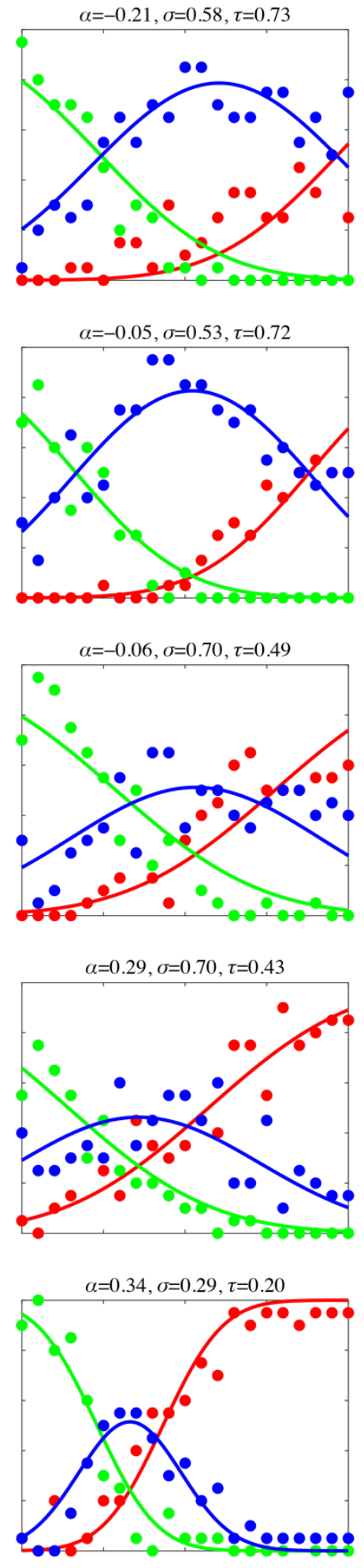
$20 \%$

S20

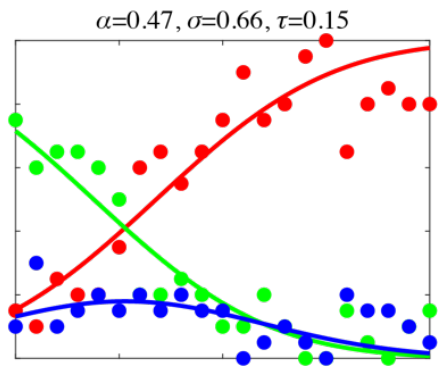

S23

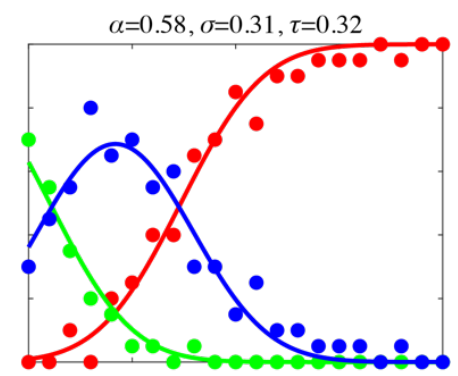

S24

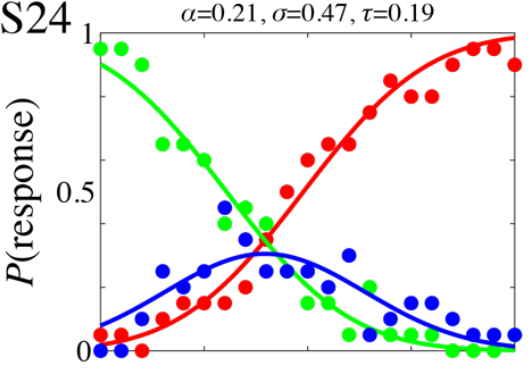

S31

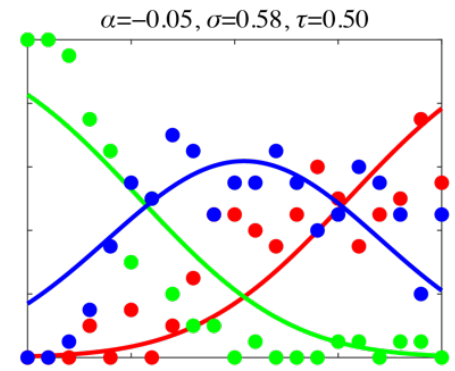

S32

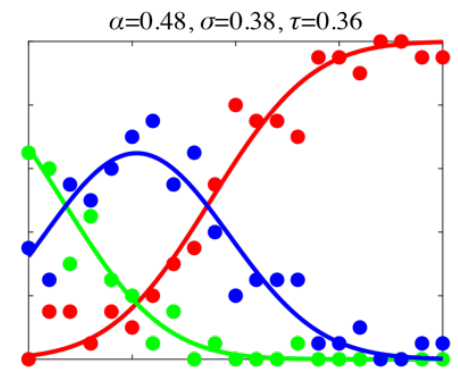

$25 \%$
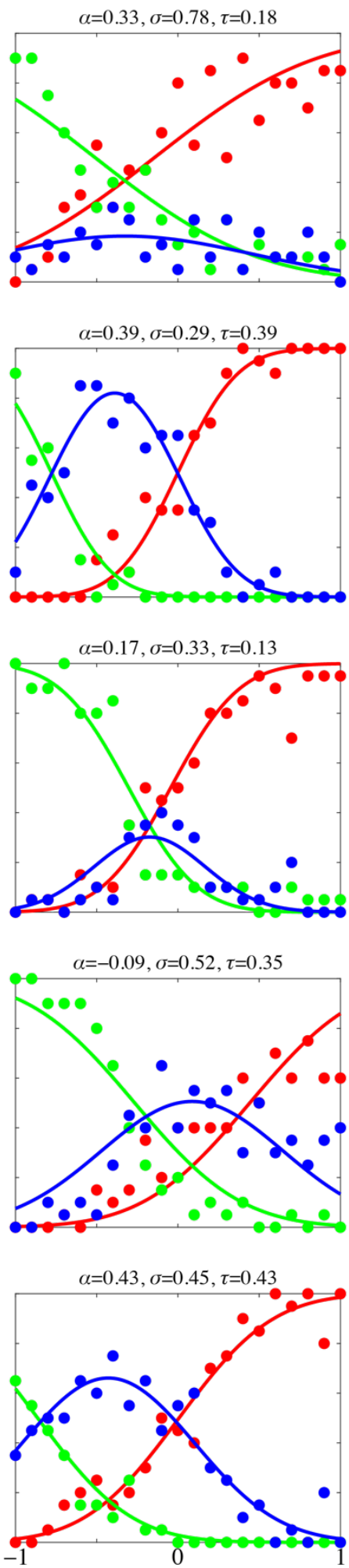

$\log \left(c_{1} / c_{2}\right)$
$30 \%$
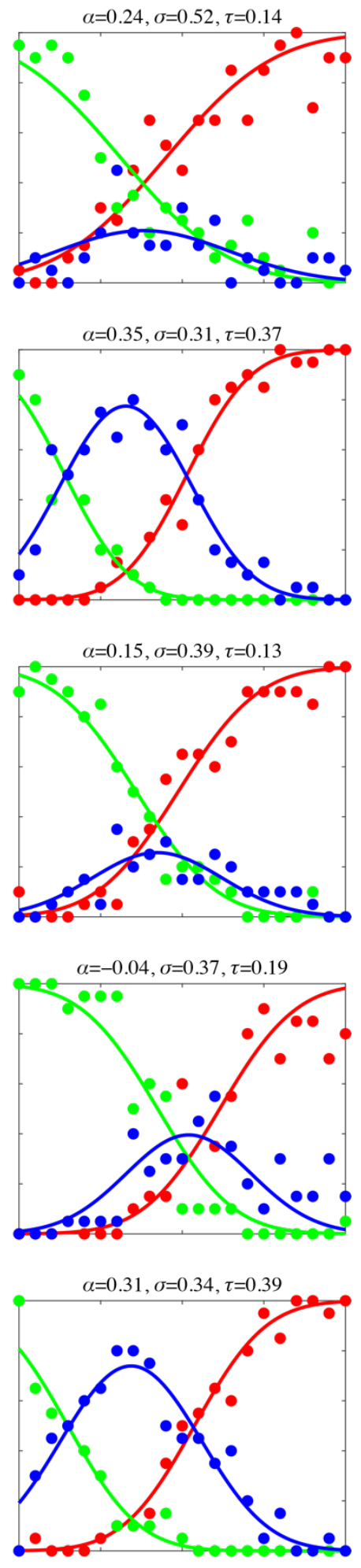
Excluded subjects
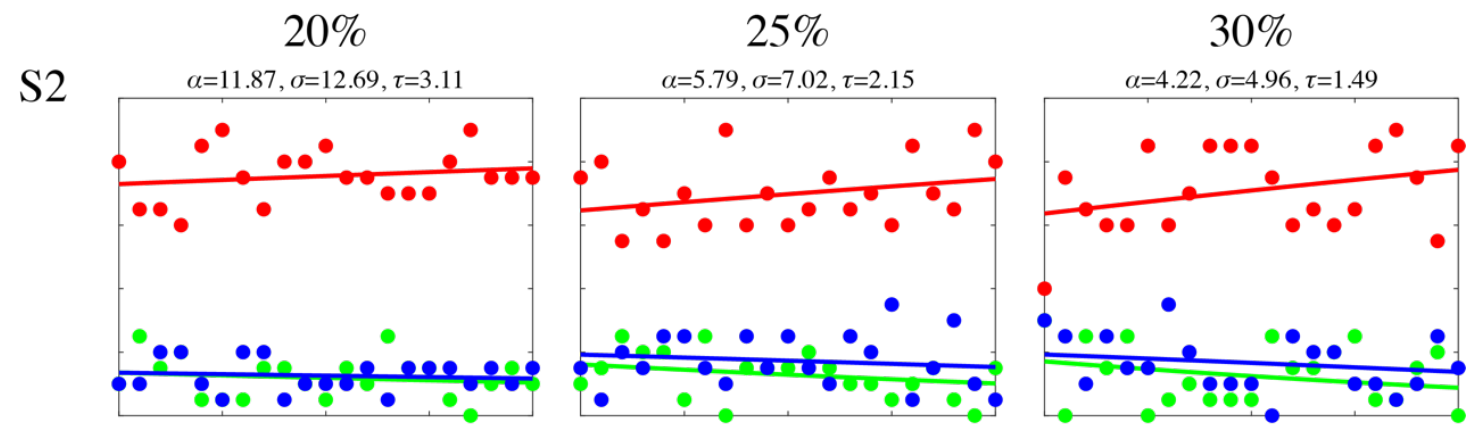

S3
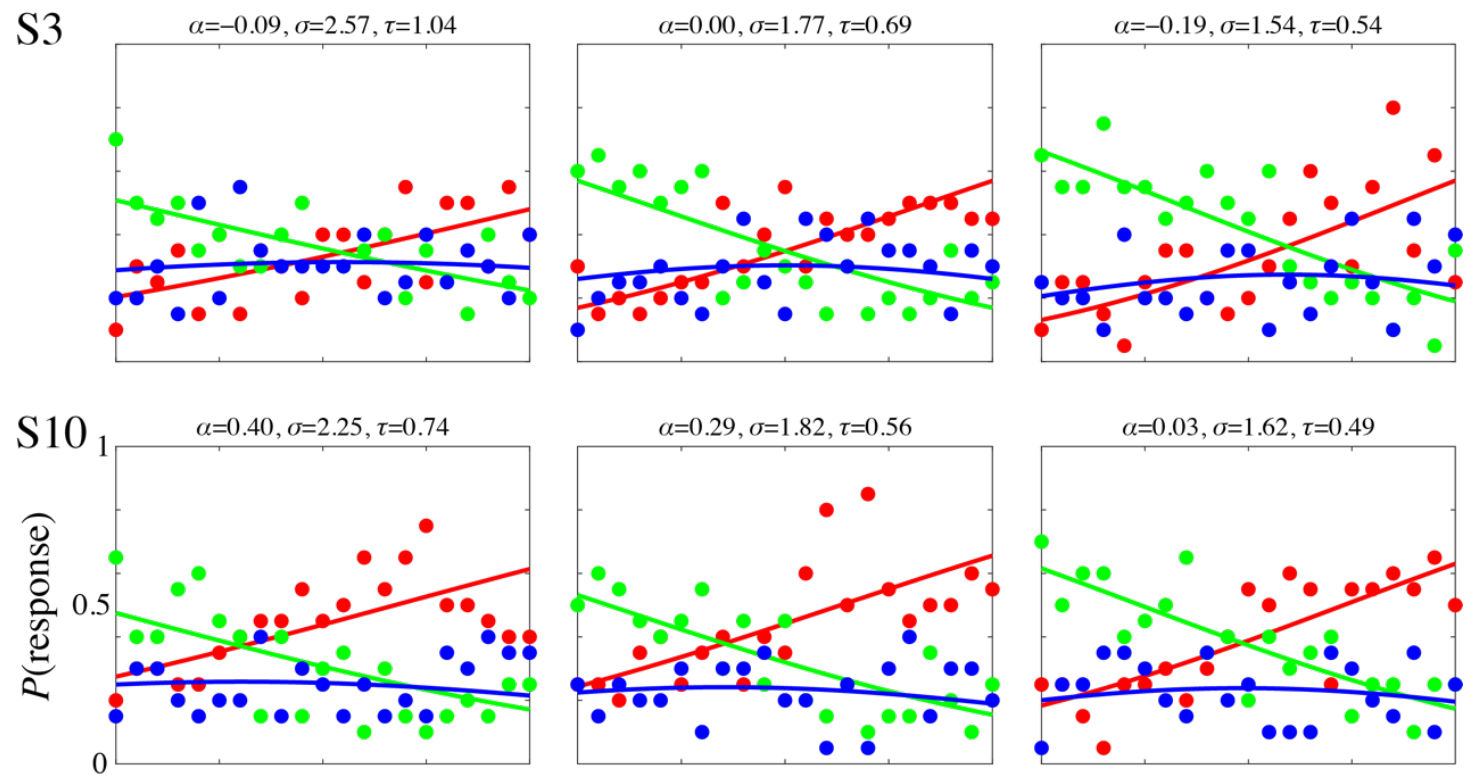

S19
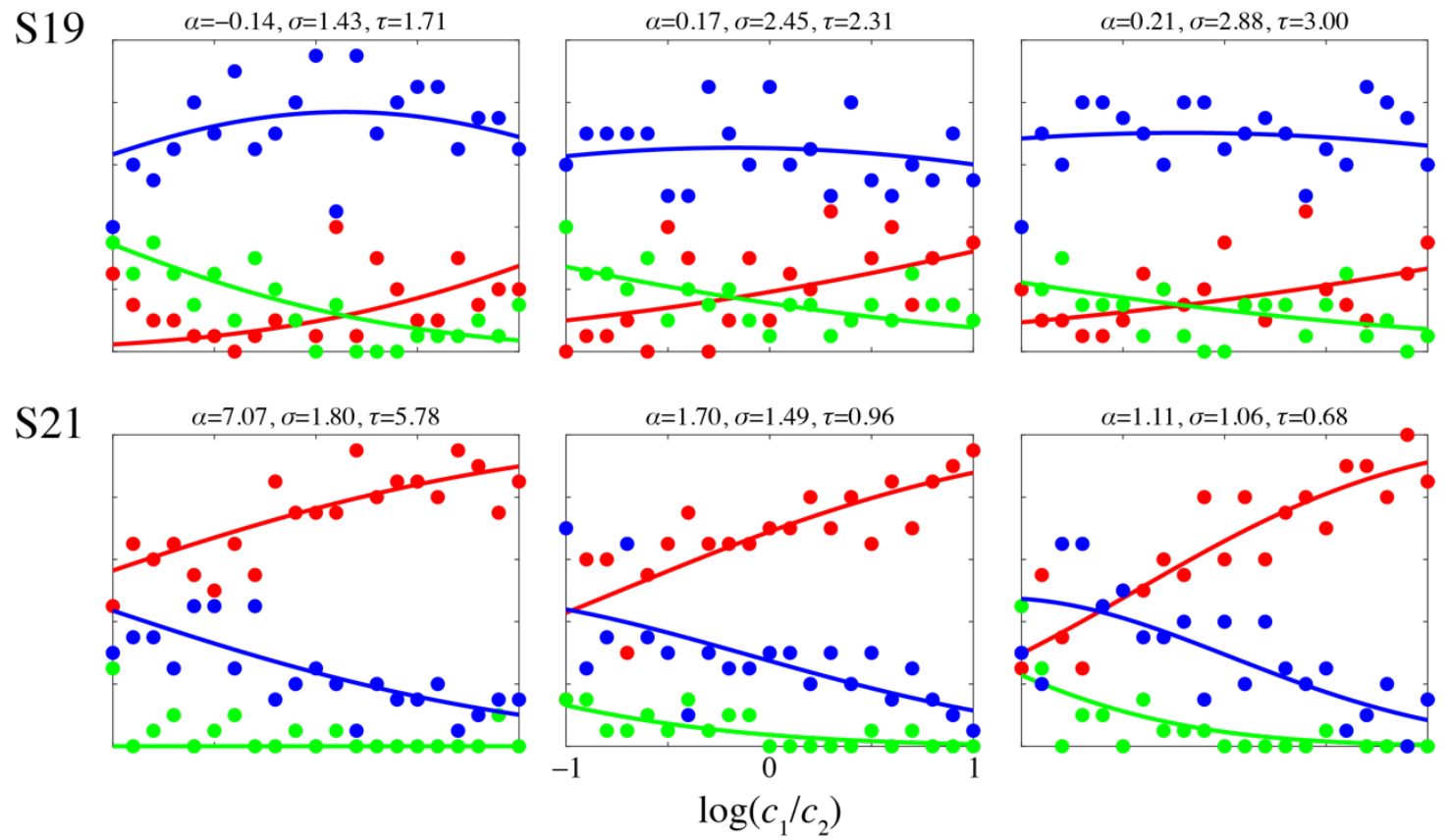
Excluded subjects

$20 \%$

S22

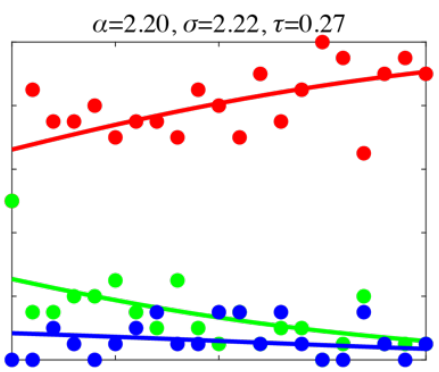

S25
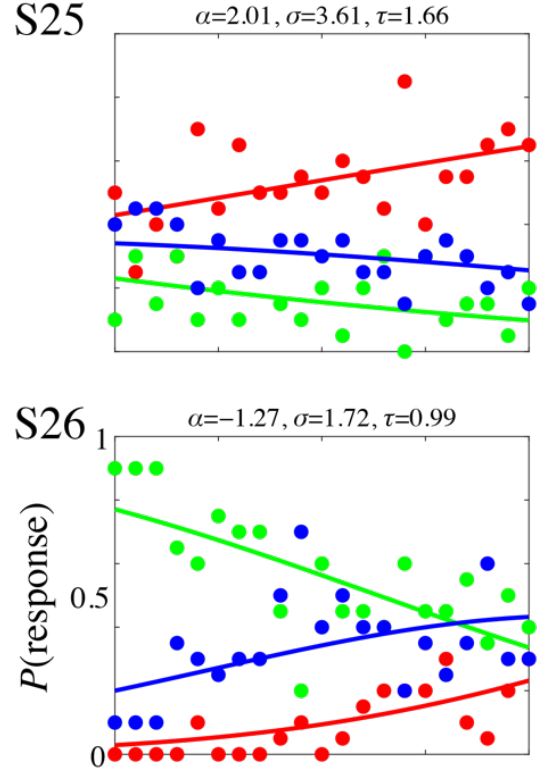

S27

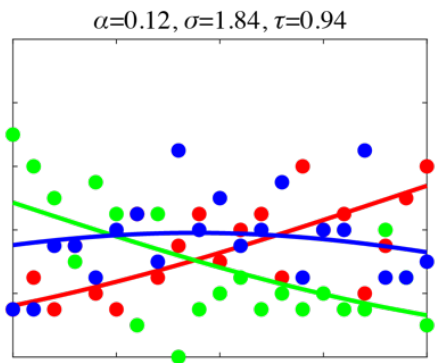

S28

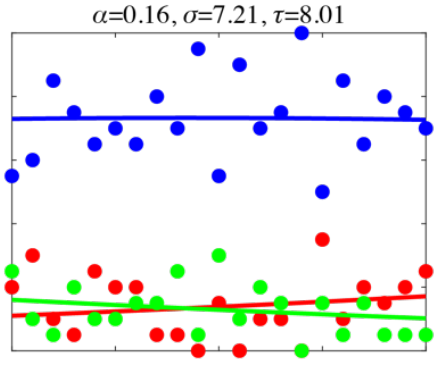

$25 \%$
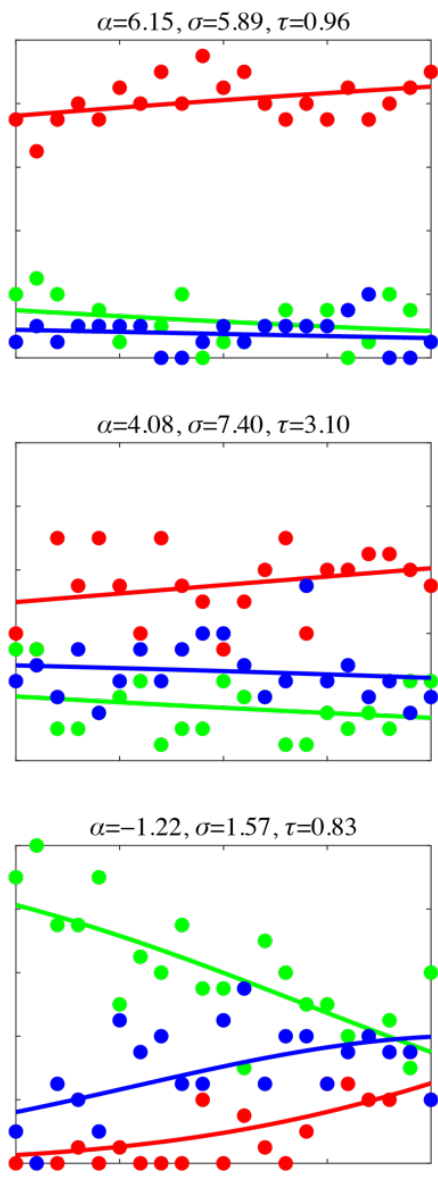

$\alpha=0.05, \sigma=1.57, \tau=0.79$

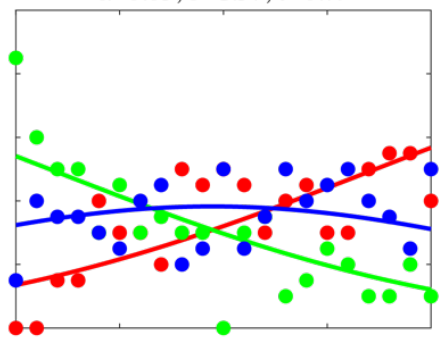

$\alpha=0.36, \sigma=7.64, \tau=7.92$

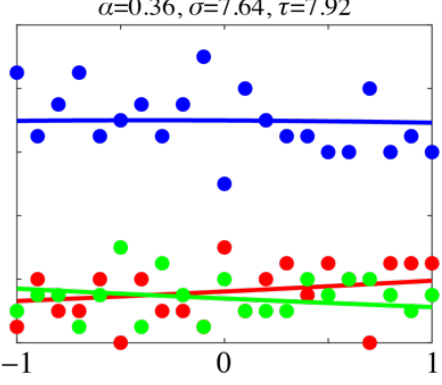

$\log \left(c_{1} / c_{2}\right)$
$30 \%$

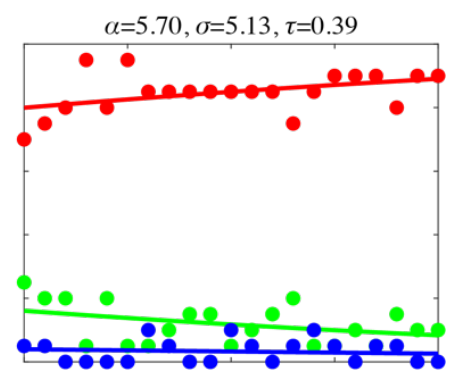

$\alpha=0.79, \sigma=2.67, \tau=1.02$

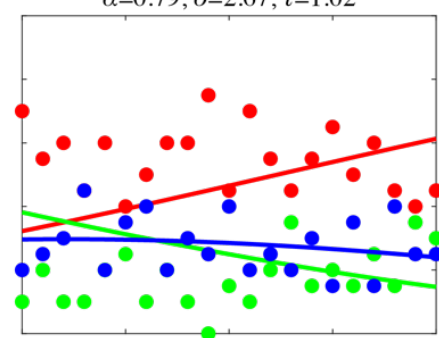

$\alpha=-0.75, \sigma=1.12, \tau=0.37$

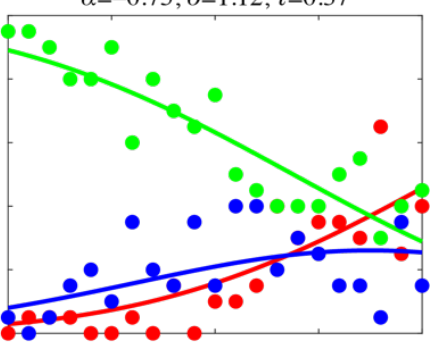

$\alpha=0.07, \sigma=1.15, \tau=0.45$

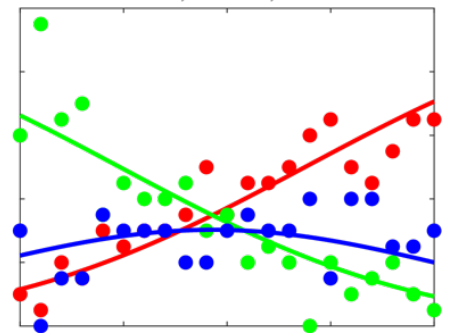

$\alpha=1.44, \sigma=17.96, \tau=18.24$

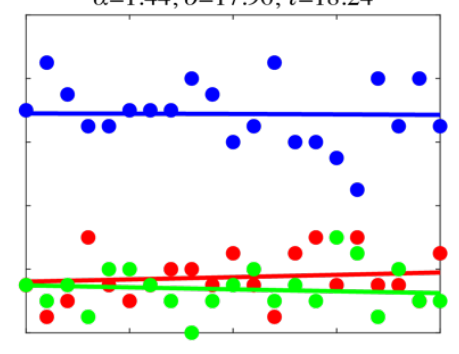

